Bull. Mater. Sci., Vol. 8, No. 2, May 1986, pp. 247-251. (C) Printed in India.

\title{
Computer simulation of dislocation-carbon interaction in nickel
}

\author{
B PURNIAH and S SRINIVASAN \\ Indira Gandhi Centre for Atomic Research, Kalpakkam 603 102, India
}

\begin{abstract}
An atomistic computer model based on a rigid boundary method has been developed to estimate the interaction energy between a single edge dislocation and an impurity carbon interstitial in nickel. The simulation was carried out using the static relaxation technique on a total of 600 atoms, using an appropriate host-host pair potential. The impurityhost potential has been chosen to be a cubic function in this calculation. In the absence of the dislocation the dipole tensor for the octahedral site is seen to be spherical. Two impurity positions, one above and one below the slip plane were investigated. The carbon-dislocation interaction energies obtained were $1.4 \mathrm{eV}$ and $0.7 \mathrm{eV}$ respectively for the two impuxity positions. The dipole tensor for the impurity in the presence of the dislocation was found to be non-diagonal.
\end{abstract}

Keywords. Computer simulation; impurity defeets; dislocation; lattice statics; interaction.

\section{Introduction}

Many important physical and technological phenomena occur as a direct consequence of the interaction between impurity point defects and dislocations. A well-known example is that of strain aging which involves the migration of interstitial impurities to dislocations. This interaction can be studied very effectively by internal friction experiments (Nowick and Berry 1972). The quantitative interpretation of internal friction experiments needs the estimation of the dipole tensor which is a measure of the elastic distortion caused by the defect in question. In this paper we describe such a calculation for a straight edge dislocation and a carbon impurity interstitial in foc nickel. The simulation is carried out in two steps. Section 2 describes the choice of an interatomic potential. Section 3 describes the simulation of an edge dislocation and $\$ 4$ describes the simulation of the dislocation-point defect interaction.

The simulation of an edge dislocation described here is based on a rigid boundary method (Bullough and Tewary 1979). To obtain the atomic configurations associated with the straight edge dislocation, a rectangular parallelopiped of freely interacting atoms was set up. The defect configuration is introduced at the centre of this crystallite which has two regions. In the inner region (region I), the atoms are allowed to freely relax to the equilibrium positions via a given nonlinear potential. In the outer region (region II), the atoms are constrained to remain in positions given by the linear elasticity theory. Region I was chosen to be sufficiently large compared to region II such that the influence of the surface or boundary effects are minimized. Some improved methods involving flexible boundaries (Hoagland et al 1976) have been developed and used in dislocation simulation. However, these flexible boundary methods cannot be used for a combined point defect-dislocation model.

There are two main techniques followed in literature to relax the atoms in Region I. These are the static and the dynamic relaxation techniques. The static method which is 
basically a gradient method is used here to minimize the total energy of this region (Sahu et al 1980). To determine the interaction energy, we need to evaluate the difference in the energy of formation of the point defect in a perfect and a lattice with a dislocation. This is accomplished by a separate evaluation of the energy of the dislocation and the point defect-dislocation combination.

\section{Interatomic potentials}

The interatomic potential is of crucial importance in atomistic models. They can be classified into two broad categories, namely the empirical potentials and the pseudopotentials. The pseudopotential method, which has been successful in predicting various physical properties of the perfect lattice has been shown to be inadequate for defects such as vacancies, dislocations, surfaces etc., which produce large inhomogeneities in the electron distribution. Empirical potentials are, however, more suited for defect studies (Johnson 1973). The simulation of dislocations in fcc metals require pair potentials which incorporate stacking-fault energies. We have used such a potential developed by Baskes and Melius (1979) for the host lattice where the cut-off distance for the potential is taken to be between the third and fourth neighbour. The functional form of the potential is a cubic spline between a number of fixed nodal points. The impurity-host potential was chosen to be a cubic function (Johnson et al 1964). The general shape was similar to the host-host potential i.e. a strong repulsion at close distances, a minimum at some intermediate distance and going to zero with zero slope at a cut-off distance. The potential parameters were determined with the help of experimental quantities like the carbon atom migration energy, activation volume and carbon-vacancy binding energy.

\section{Simulation of edge dislocation}

The edge dislocation studied in this case lies along [112] direction. Its Burger's vector was of the type $a / 2\langle 110\rangle$ (see figure 1 ). The faces of the crystallite were appropriate $\{110\},\{112\}$, and $\{111\}$ crystallographic planes and the dislocation was arranged to lie through the centre of the assembly and orthogonal to the two $\{112\}$ faces. In the direction of the dislocation line, specifically the [112] direction ( $y$-axis), the assembly was only six lattice planes thick and periodic boundary conditions were imposed across the two end (112) faces (Cotterill and Doyama 1967). In the other two orthogonal directions ([110]- the $x$ direction and [111]- the $z$ direction) the assembly was made up of $49\{110\}$ lattice planes and $19\{111\}$ planes. The parallelopiped was deliberately extended in the $x$-direction to accommodate the dissociation into Shockley partials of the glissile edge dislocation. The initial defect configuration was achieved by first imposing the appropriate anisotropic elastic displacements on all the atoms in the crystallite. This configuration is designated as the elasto-atomic model.

Next, the atoms in region I, numbering approximately 600 , were allowed to relax towards equilibrium under the influence of the host pair potential already described. During the simulation of the undissociated dislocation (also called complete dislocation) the atoms were not permitted to relax in a direction parallel to the dislocation line. The strain energy of a dislocation inside a certain radius is given by 


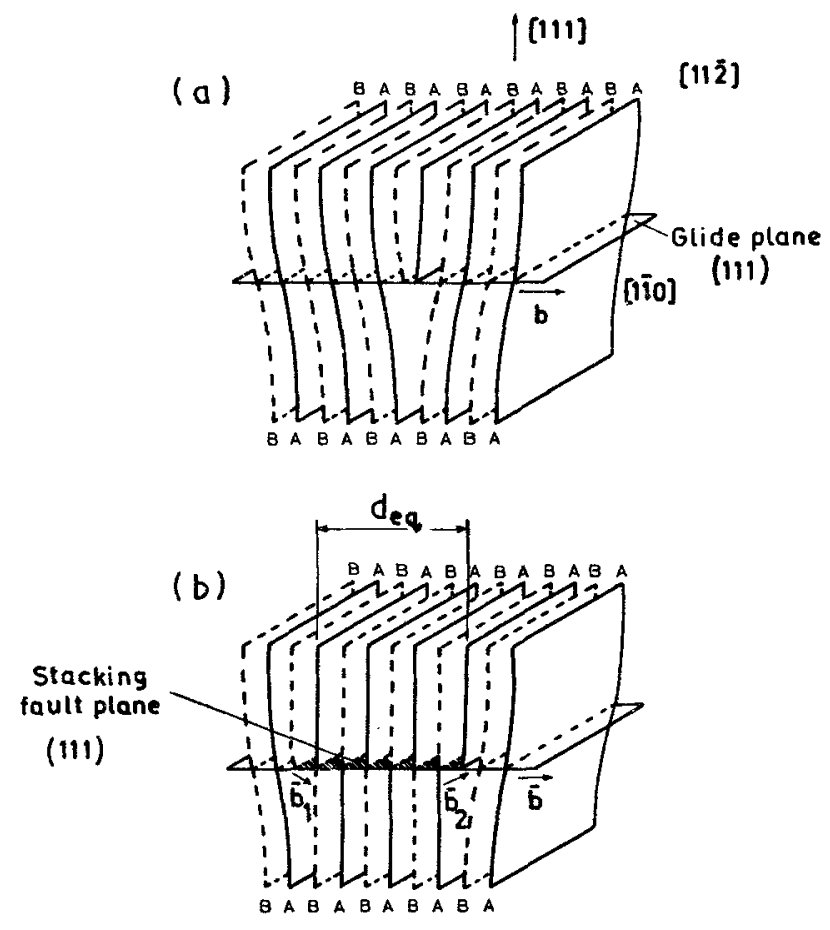

Figure 1. Spatial representation of an edge dislocation with a Burger's vector $a / 2[1 \overline{1} 0]$ in the foc structure. (a) Complete edge dislocation. (b) Dissociated edge dislocation. $\left(\bar{b}, \bar{b}_{1}\right.$ and $\bar{b}_{2}$ indicate the Burger's vector of the complete and the two partial dislocations).

$E_{d}=1 / 2 \Sigma_{i}^{n}\left(E_{i}-E_{0}\right)$, where $n$ is the number of atoms inside that radius, $E_{i}$ is the energy of the $i$ th atom after relaxation and $E_{0}$ in the perfect lattice. The factor $1 / 2$ is required because each interatomic bond is counted twice in the calculation. Figure 2 shows the energies of the elasto-atomic model and the complete edge dislocation plotted as a function of distance.

It is known in foc metals that the edge dislocation dissociates into a pair of partial dislocations separated by an intrinsic stacking-fault on the (111) plane (Heidenreich and Shockley 1948). To study this dissociation, the atoms in region I were also allowed to relax parallel to the dislocation line. The parallelopiped was extended to 49 planes in the $x$-direction (as already stated) to accommodate the splitting of the dislocation into partials leading to a stacking-fault along this direction. The final positions attained by the atoms in complete dislocation were used as the initial positions for the case of the dissociated dislocation. It was found that the complete dislocation splits into partials separated by a distance of $8 \mathrm{~b}$. The energy of the dissociated dislocation is also plotted in figure 2 . It can be seen that the energy of the dissociated dislocation is indeed less than that of the complete dislocation.

\section{Dislocation-carbon interaction}

To obtain the interaction energy between the dislocation and carbon impurity, it was necessary to extend the parallelopiped in the $y$-direction and drop the periodic 


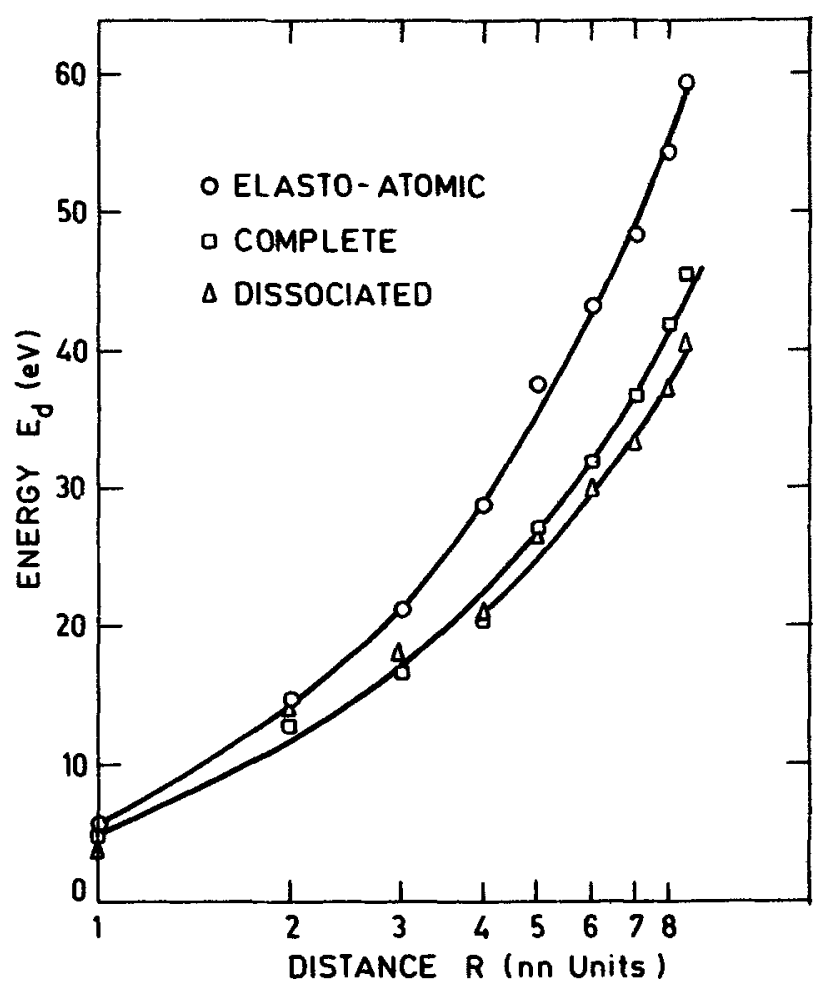

Figure 2. The energy as a function of the radius from the centre of a edge dislocation shown for the three cases, elasto-atomic, complete and dissociated dislocation.

boundary conditions on these faces. The crystallite was made 30 planes thick as against the six originally taken. Of this assembly, the central region containing 627 atoms was allowed to relax. The boundary atoms were held in their previous relaxed dissociated dislocation positions and the position of the point defect (in this case the carbon impurity in octahedral site) relative to the dislocation was varied. Two impurity positions were chosen. One position at $(3,-3,1.5)$ was above the slip plane and the other at $(3,-3,-1.5)$ was below the slip plane. The co-ordinates are with reference to the dislocation centre located at $(0,0,0)$. The carbon interstitial formation energy in the perfect lattice was obtained using a point defect simulation program (Sahu et al 1980).

\section{Results and discussion}

The results of the simulation are summarized in table 1 . The edge dislocation lying along [112] direction was found to dissociate into partials when the atoms in region $I$ were allowed to relax freely in all three directions. The equilibrium distance of separation between the partials was found to be $8 \mathrm{~b}$. For the two impurity configurations, one above and one below the slip plane, the formation energy, interaction energy and dipole tensor were obtained. A few points can be seen from the table. The (undersized) carbon atom prefers to sit above the slip plane as is known from the 
Table 1. Values of carbon impurity formation energy, interaction energy, dipole tensor and stacking-fault distance obtained from computer simulation of an edge dislocation in $\mathrm{Ni}$.

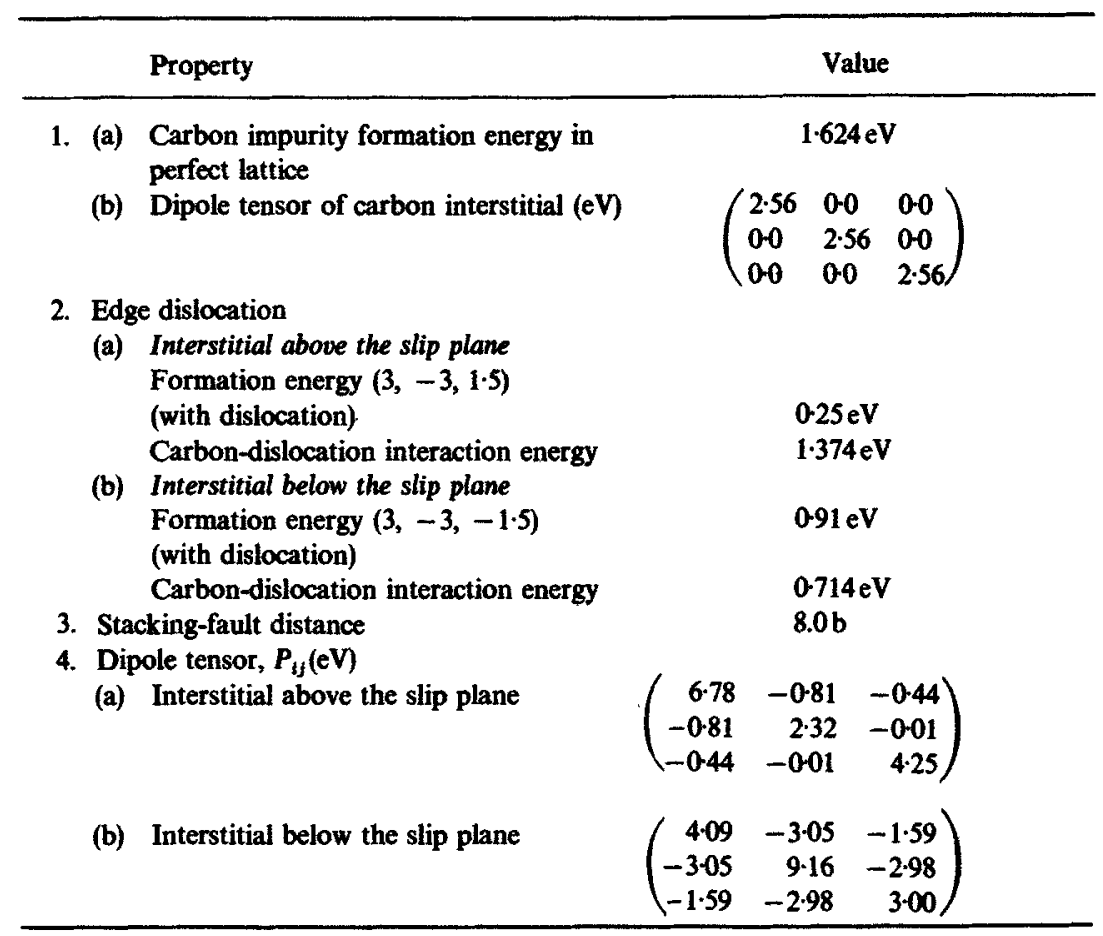

Cottrell formula using linear elasticity theory. Further, the dipole tensor which is spherical in the absence of the dislocation has a monoclinic symmetry in the vicinity of the dislocation. This is true both above and below the dislocation.

\section{References}

Abrahamson A 1969 Phys. Rev. 17876

Baskes M I and Melius C F 1979 Phys. Rev. 203197

Bullough R and Tewary V K 1979 in Dislocations in solids (ed.) F R N Nabarro (New York: North-Holland) Vol. 2, p. 1

Cotterill R M J and Doyama M 1967 in Lattice defects and their interactions (ed.) R R Hasiguti (New York: Gordon and Breach), p. 20

Heidenreich R O and Shockley W 1948 Report on Conference in Strength of Solids, p. 57

Hoagland R G, Hirth J P and Gehlen P C 1976 Philos. Mag. 34413

Johnson R A 1973 J. Phys. F3 295

Johnson R A, Dienes G J and Damask A C 1964 Acta Metall. 121215

Nowick A S and Berry B S 1972 Anelastic relaxation in crystalline solids (New York: Academic Press)

Sahu H K. Srinivasan S and Krishan K 1980 Pramana (J. Phys.) 15189 